\title{
Forkhead box C1 promoter upstream transcript, a novel long non-coding RNA, regulates proliferation and migration in basal-like breast cancer
}

\author{
JUNTAO LIU ${ }^{1 *}$, LEI SHEN ${ }^{2 *}$, JIE YAO $^{3,4^{*}}$, YI LI $^{4}$, YONGCHENG WANG ${ }^{5}$, \\ HONG CHEN $^{6}$ and PEILIANG GENG ${ }^{4}$
}

\begin{abstract}
${ }^{1}$ Department of General Thoracic Surgery, Affiliated Hospital of Logistics University of Chinese People's Armed Police Forces, Tianjin 300162; ${ }^{2}$ Department of Endocrinology, Chinese PLA General Hospital, Beijing 100853; ${ }^{3}$ Department of Oncology, PLA Center Hospital, Wuhan, Hubei 430000; ${ }^{4}$ Oncology Department, Cancer Center, Division of Internal Medicine, Chinese PLA General Hospital, Beijing 100853; ${ }^{5}$ Orthopedics Department, Institute of Orthopedics, Chinese PLA General Hospital, Beijing 100853; ${ }^{6}$ Department of Oncology, Kunming General Hospital of Chendu Military Command, Kunming, Yunnan 650032, P.R. China
\end{abstract}

Received February 18, 2014; Accepted November 14, 2014

DOI: $10.3892 / \mathrm{mmr} .2014 .3089$

\begin{abstract}
Recent studies have shown that long non-coding RNAs (lncRNAs) have crucial regulating roles in carcinogenesis. Forkhead box $\mathrm{C} 1$ (FOXC1) is an important cancer-associated gene in basal-like breast cancer (BLBC). In the present study, a novel lncRNA, FOXC1 promoter upstream transcript (FOXCUT) was investigated in BLBC patients using polymerase chain reaction analysis. The results showed that the expression of FOXCUT and FOXC1 were positively correlated. When the expression of FOXCUT was downregulated by small interfering RNA, the expression of FOXC1 was similarly reduced. Furthermore, in MDA-MB-231 and MDA-MB-468 breast cancer cells, knockdown of FOXCUT markedly inhibited cell proliferation and migration in vitro. In conclusion, FOXCUT lncRNA may be functionally involved in the tumor progression of BLBCs through the regulation of its paired mRNA, FOXC1, demonstrating that FOXCUT may serve as a novel biomarker and therapeutic target in BLBCs.
\end{abstract}

Correspondence to: Dr Peiliang Geng, Oncology Department, Cancer Center, Division of Internal Medicine, Chinese PLA General Hospital, 28 Fuxing Road, Beijing 100853, P.R. China

E-mail: libracheria@yahoo.com

Professor Hong Chen, Department of Oncology, Kunming General Hospital of Chendu Military Command, 212 Daguan Road, Kunming, Yunnan 650032, P.R. China

E-mail: capricorn0713@hotmail.com

*Contributed equally

Key words: basal-like breast cancers, long non-coding RNA, forkhead box $\mathrm{C} 1$, proliferation, migration

\section{Introduction}

Basal-like breast cancers (BLBCs) are aggressive malignancies that express the gene signatures of basal/myoepithelial cells in mammary glands. In human breast cancers, the basal-like subtype has been identified as a distinct entity with a poor prognosis. Due to their underexpression of estrogen receptor (ER), progesterone receptor (PR) and HER2, BLBCs are unlikely to respond to the current targeted systemic therapy (1-3).

To overcome the challenges in the treatment of BLBCs, a novel avenue of investigation into the molecular basis for this disease is urgently required. Recently, a number of studies have demonstrated that long non-coding RNAs (lncRNAs) have pivotal roles in the origination and progression of certain types of cancer (4-6). While only a few studies on lncRNAs in BLBCs have been reported, targeting lncRNAs with critical regulating activities in BLBCs is a promising therapeutic strategy for the future.

lncRNAs are RNA transcripts with no protein-coding potential that are $>200$ bases in length, which have been identified as high level regulators with multiple molecular regulating mechanisms in gene networks (7-9). A recent study revealed that numerous IncRNAs functionally contact their adjacent mRNAs and take on the form of 'IncRNA-mRNA pairs' in the regulatory network (10).

Through preliminary bioinformatics analysis (http://genome.ucsc.edu/), the present study found the novel IncRNA TCONS_00011636, which is located at 6p25 and is transcribed from the upstream side of the FOXC1 promoter. Therefore, it was named as FOXC1 promoter upstream transcript (FOXCUT) by our group. FOXC1 is an important transcriptional factor regulating a variety of biological processes, including embryogenesis, tumorigenesis and epithelial-mesenchymal transition (EMT) (11-13). Several recent studies have shown that a high level of FOXC1 expression correlates with poor overall survival in BLBCs, and that FOXC1 is associated with aggressive phenotypes and increased cell proliferation 
Table I. Primers for real time polymerase chain reaction analysis.

\begin{tabular}{lcc}
\hline Gene name & \multicolumn{1}{c}{ Forward } & \multicolumn{1}{c}{ Reverse } \\
\hline$\beta$-actin & 5'-CCACTGGCATCGTGATGGA-3' & 5'-CGCTCGGTGAGGATCTTCAT-3' \\
FOXC1 & 5'-GGCGAGCAGAGCTACTACC-3' & 5'-TGCGAGTACACGCTCATGG-3' \\
FOXCUT & 5'-GTCGCACCGATGACTAACG-3' & 5'-GCCCTGAAAGCCGAACTG-3' \\
\hline
\end{tabular}

Table II. Sequences for small interfering RNA analysis.

\begin{tabular}{lcc}
\hline Gene name & Sense (5'-3') & Antisense (5'-3') \\
\hline FOXCUT si1 & 5'-GAAUGGAGAACUAAGACAAUUAUCT-3' & 5'-AGAUAAUUGUCUUAGUUCUCCAUUCGG-3' \\
FOXCUT si2 & 5'-CAGCCUCCCUCCUGUGUGUGCAGAG-3' & 5'-CUCUGCACACACAGGAGGGAGGCUGCA-3' \\
\hline
\end{tabular}

and migration in breast cancer cells (14-16). In other types of malignant tumors, including pancreatic cancer, non-small cell lung cancer and hepatocellular carcinoma $(12-14,17,18)$, overexpression of $\mathrm{FOXC1}$ is strongly correlated with poor prognosis of the patients. FOXC1 is now recognized as an important cancer biomarker in BLBCs $(15,16)$. However, the expression and function of FOXCUT IncRNA in BLBCs and its association with the adjacent mRNA FOXC1 remains to be determined.

The aim of the present study was to investigate the expression profile of FOXCUT IncRNA in breast cancer tissues and the functional role of FOXCUT in MDA-MB-231 and MDA-MB-468 human BLBC cells in vitro.

\section{Materials and methods}

Patient samples. A total of 55 specimens were collected from 55 patients previously diagnosed with primary breast cancers at the PLA General Hospital (Beijing, China) between 2007 and 2013. Clinical classification was performed by immunohistochemical studies for ER, PR, HER2, cytokeratin $5 / 6$ and EGFR (19,20). The series included examples from each of the molecular subtypes based on their immunohistochemical surrogate: 16 luminal A-like (ER+/PR+/HER2-), 8 luminal B-like (ER+/PR+/HER2+), 6 HER2-enriched (ER-/PR-/HER2+) and 25 basal-like (ER-/PR-/HER2+/CK5/6+ or EGFR+). The utilization of tumor material for research was approved by the ethical committee of PLA General Hospital and written informed consent was obtained from the patients or their families.

Cell line and cell culture. The human BLBC cell lines MDA-MB-231 and MDA-MB-468 were purchased from the American Type Culture Collection (Manassas, VA, USA). The MDA-MB-231 cells were incubated in Dulbecco's modified Eagle's medium (Hyclone, Logan, UT, USA) containing 10\% fetal bovine serum (FBS; Invitrogen, Carlsbad, CA, USA), and MDA-MB-468 cells were cultured in RPMI-1640 medium (Hyclone) supplemented with $10 \% \mathrm{FBS}$ at $37^{\circ} \mathrm{C}$ with $5 \% \mathrm{CO}_{2}$.

Reverse transcription-quantitative polymerase chain reaction $(R T-q P C R)$. Total RNA was extracted from breast cancer tumor tissues, matched adjacent normal tissues and breast cancer cells using the TRIzol Total RNA reagent (Invitrogen Life Technologies, Carlsbad, CA, USA). The primers were obtained from Sheng Gong (Shanghai, China) and the sequences are presented in Table I. RT-qPCR was performed using the SYBR PrimeScript RT-PCR kit (Takara, Ohtsu, Japan) in an Applied Biosystems 7500 Fluorescent Quantitative PCR System (Applied Biosystems, Foster City, CA, USA). The reaction mixtures were incubated at $95^{\circ} \mathrm{C}$ for $30 \mathrm{sec}$, followed by 40 amplification cycles of $95^{\circ} \mathrm{C}$ for $5 \mathrm{sec}$ and $60^{\circ} \mathrm{C}$ for $34 \mathrm{sec}$. The comparative $\mathrm{Ct}$ method was used to quantify relative expression of mRNA and lncRNA. Expression levels of housekeeping gene $\beta$-actin were used to normalize gene-of-interest expression. The expression levels of a target gene in a patient were calculated as the ratio of the target expression levels in tumor tissue to the target expression levels in non-tumorous tissue $(\mathrm{T} / \mathrm{N})$.

Transfection of siRNA. The siRNA sequences were obtained from GenePharma (Shanghai, China), including one negative control siRNA (NC siRNA) sequence and two FOXCUT siRNA sequences. The target sequences are presented in Table II. siRNA transfection was performed with X-tremeGENE transfection reagent (Roche, Mannheim, Germany). In brief, $\sim 5 \%$ cells were plated in each well of 12 -well plates at least $24 \mathrm{~h}$ prior to transfection to achieve $30-50 \%$ confluency. siRNA transfection was then performed with X-tremeGENE transfection reagent (Roche) according to the manufacturer's instructions. Two days post-transfection, RNA isolation, cell proliferation assay, scratch wound healing assay and matrigel invasion assays were performed.

Cell proliferation assay. Following transfection, cell proliferation was assessed by a CellTiter $96^{\circledR}$ Aqueous 3-(4,5-dimethylthiazol-2-yl)-5-(3-carboxymethoxyphenyl)-

2-(4-sulfophenyl)-2H-tetrazolium (MTS) assay kit (Promega) according to the manufacturer's instructions. MDA-MB-231 and MDA-MB-468 cells (2,000 cells per well) in each group were plated in 96-well plates. MTS reagent $(20 \mu \mathrm{l})$ was added to each well containing $100 \mu \mathrm{l}$ culture medium. The plate was incubated for $2 \mathrm{~h}$ at $37^{\circ} \mathrm{C}$ in a humidified, $5 \% \mathrm{CO}_{2}$ atmosphere. The plate was read at a wavelength of $490 \mathrm{~nm}$ using a SpectraMax M2 plate reader (Molecular Devices, Sunnyvale, CA, USA). 
A

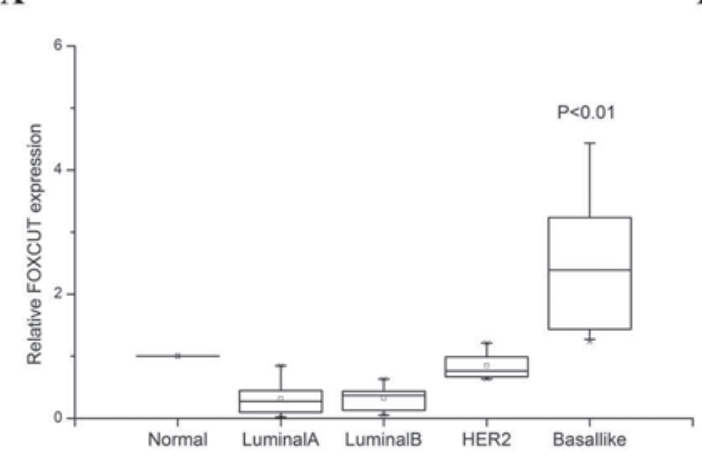

B

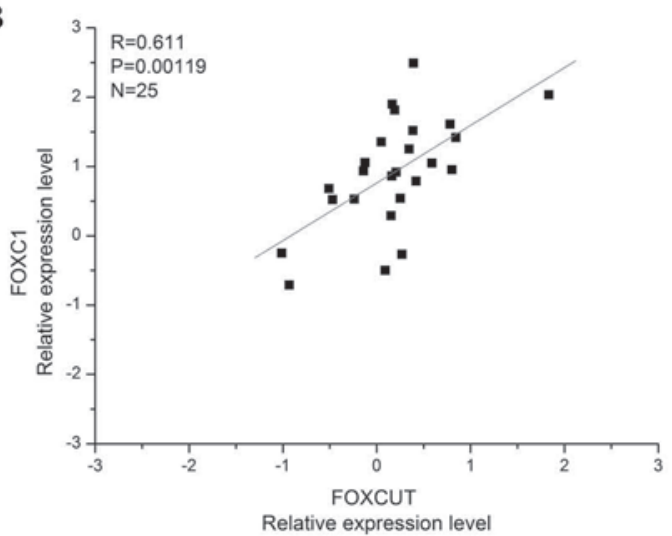

Figure 1. FOXCUT long non-coding RNA expression levels were analyzed by RT-qPCR in 55 breast cancer tissue samples. (A) Expression levels of FOXC1 in breast cancer were significantly higher than those in adjacent normal tissues by RT-qPCR. (B) The expression levels of FOXC1 were significantly correlated with those of FOXCUT 1ncRNA $(\mathrm{R}=0.611 \mathrm{P}<0.01)$. RT-qPCR, reverse transcription quantitative polymerase chain reaction, FOXCUT, forkhead box $\mathrm{C} 1$ promoter upstream transcript.

Scratch wound healing assay. Prior to transfection, uniform wounds were scraped into MDA-MB-231 and MDA-MB-468 cells grown on plastic six-well plates using a pipette tip. The initial gap length $(0 \mathrm{~h})$ and the residual gap length $48 \mathrm{~h}$ after wounding were calculated from photomicrographs. Images were captured using a Olympus BX51 Clone fluorescence microscope (Olympus Corp., Tokyo, Japan).

Matrigel invasion assays. A cell invasion assay was performed using modified Boyden Chambers consisting of Transwell-precoated matrigel membrane filter inserts with 8-mm pores in 24-well tissue culture plates (BD Biosciences, Franklin Lakes, NJ, USA). Culturing medium containing 10\% FBS in the lower chamber served as the chemoattractant. Cells that had migrated through the filter were stained and counted. The average migration rate was calculated as the increasing radius of the entire cell population over time.

Statistical analysis. Differences between groups were analyzed using a Student's t-test. Correlation between gene expression levels were studied using Pearson's correlation. Statistical analyses were performed using SPSS version 18.0 (International Business Machines, Armonk, NY, USA). For all statistical analyses, $\mathrm{P}<0.05$ was considered to indicate a statistically significant difference.

\section{Results}

FOXCUT is overexpressed in BLBC tissue specimens. The FOXCUT IncRNA expression levels were assessed in a panel of paired specimens obtained from 55 patients with breast cancer using RT-qPCR. The results revealed that the FOXCUT expression levels in BLBC tumor tissues were significantly higher than those in matched non-tumorous tissues. The expression levels of FOXCUT were significantly higher in BLBCs than those in non-basal like breast cancer subtypes (Fig. 1A, $\mathrm{P}<0.01$ ). In addition, the relative expression of FOXCUT was positively correlated with that of FOXC1 in the BLBC tissue samples (Fig. 1B; $\mathrm{R}=0.611$, $\mathrm{P}<0.01)$.
FOXC1 mRNA expression is suppressed by FOXCUT siRNA in BLBC cell lines. In MDA-MB-231 and MDA-MB-468 cells, RNA interference analysis was conducted to further clarify the correlation between the expression of FOXCUT lncRNA and FOXC1 mRNA. RT-qPCR was performed to evaluate the expression levels of FOXC1 mRNA and FOXCUT 1ncRNA. The results showed that FOXCUT expression was efficiently knocked down by FOXC1 siRNA (Fig. 2, P<0.05). In addition, FOXC1 expression was downregulated in the FOXCUT siRNA1 group compared with that of the NC siRNA group (Fig. 2, $\mathrm{P}<0.05$ ). This indicated that the expression of FOXC1 mRNA may be modulated by FOXCUT IncRNA in BLBCs.

Knockdown of FOXCUT inhibits the cell proliferation ability of MDA-MB-231 and MDA-MB-468 cells. To investigate the effects of FOXCUT knockdown on the in vitro growth characteristics of the MDA-MB-231 and MDA-MB-468 BLBC cell lines, an MTS assay was performed to assess the cell proliferation ability. The results showed that cell growth was inhibited in FOXCUT siRNA groups compared with that in the NC siRNA group (Fig. 3, $\mathrm{P}<0.05$ ).

Knockdown of FOXCUT suppresses the migration ability of $M D A-M B-231$ and $M D A-M B-468$ cells. To further identify the function of FOXCUT, a scratch wound-healing assay and matrigel invasion assay were performed following siRNA transfection. The results showed that the migration capacity of the MDA-MB-231 and MDA-MB-468 cells was markedly inhibited by FOXCUT siRNA (Fig. 4, P<0.05). The results of the FOXCUT knockdown concurred with the effects of FOXC1 knockdown on the in vitro growth characteristics of MDA-MB-231 and MDA-MB-468 as shown above.

\section{Discussion}

Advances in high-throughput technologies have resulted in the biological classification of breast cancer into subtypes with distinct gene expression profiles, and BLBC is the most aggressive subtype, with a unique gene-expression pattern of basal/myoepithelial cells characteristics (1-3). 
A

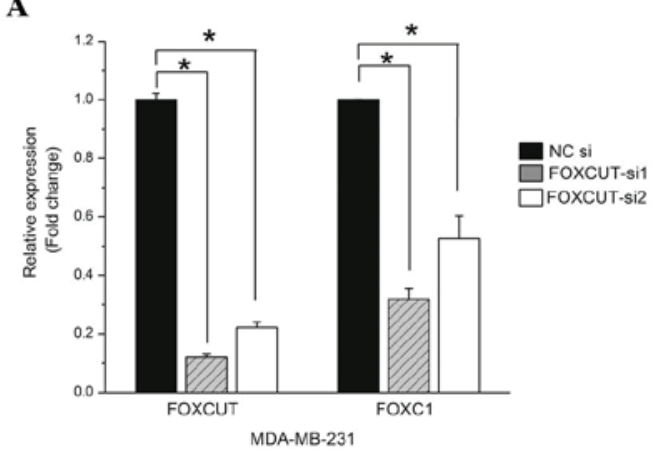

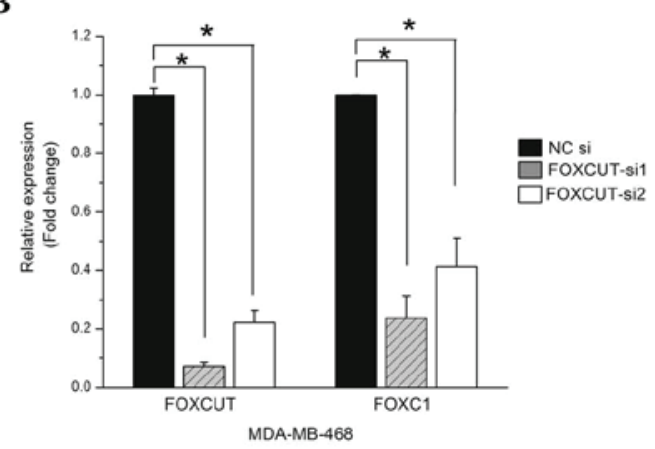

Figure 2. The expression levels of FOXC1 mRNA and FOXCUT long non-coding RNA in (A) MDA-MB-231 and (B) MDA-MB-468 cells after siRNA transfection. The results showed that the expression levels of both FOXCUT and FOXC1 were significantly knocked down in FOXCUT siRNA groups. "P<0.05 compared with the control. FOXCUT, forkhead box C1 promoter upstream transcript; siRNA, small interfering RNA; NC, negative control.
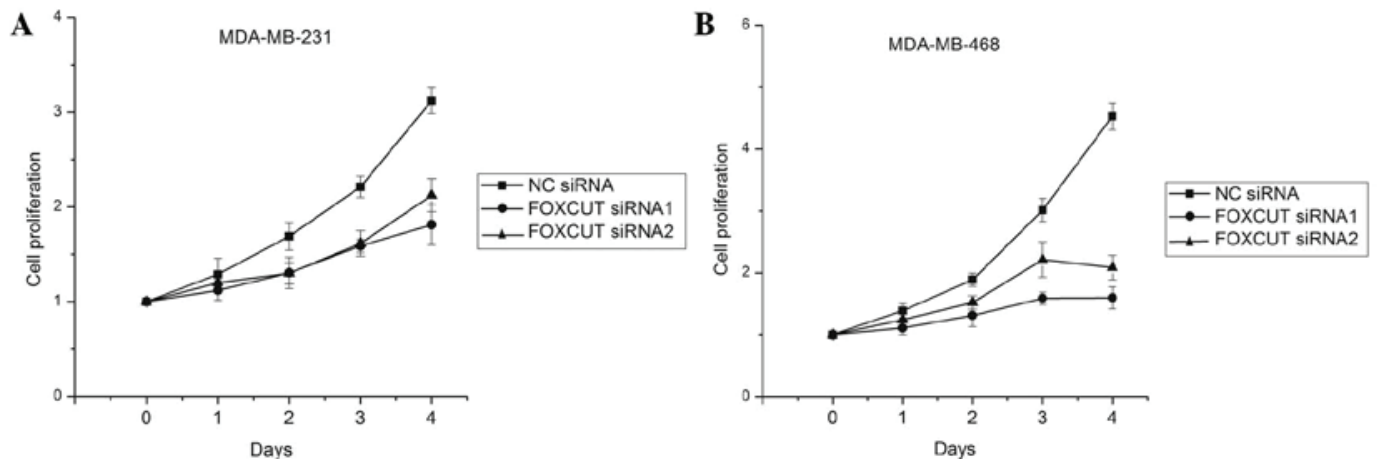

Figure 3. Effects of FOXCUT knockdown on the proliferation of MDA-MB-231 and MDA-MB-468 cells. FOXCUT siRNAs evidently reduced the growth of (A) MDA-MB-231 and (B) MDA-MB-468 compared with NC siRNA in an MTS assay ("P<0.05). FOXCUT, forkhead box C1 promoter upstream transcript; NC, negative control; siRNA, small interfering RNA; MTS, 3-(4,5-dimethylthiazol-2-yl)-5-(3-carboxymethoxyphenyl)-2-(4-sulfophenyl)-2H-tetrazolium.

A

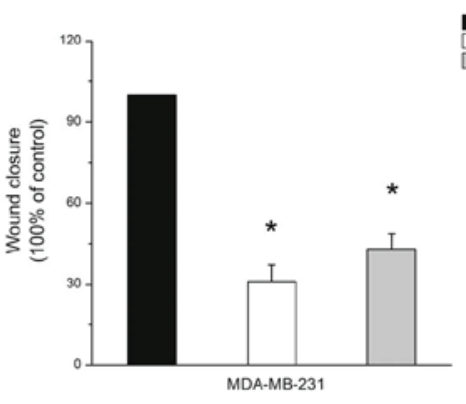

C

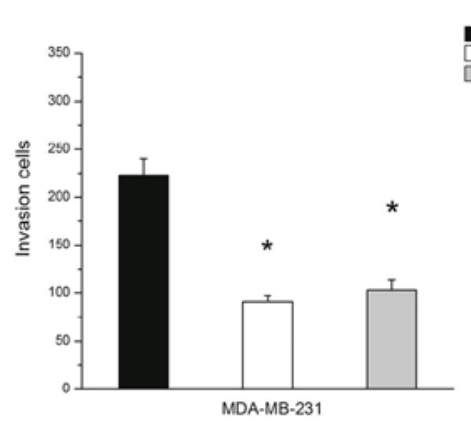

B

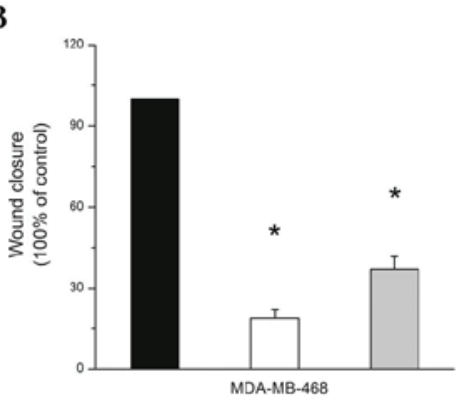

D

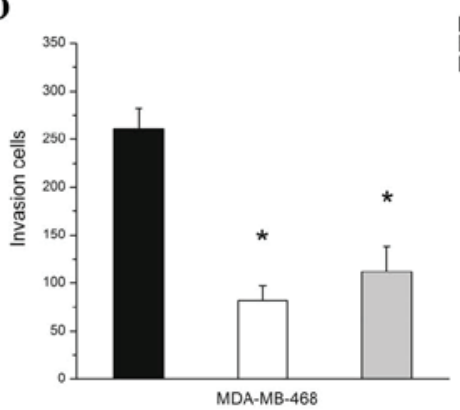

Figure 4. Effects of FOXCUT knockdown on the cell migration ability in MDA-MB-231 and MDA-MB-468. FOXCUT siRNAs evidently reduced the (A) MDA-MB-231 and (B) MDA-MB-468 cell migration compared with that of the NC siRNA control in the scratch wound healing assay. The capacity of cell invasion in the FOXCUT siRNA (C) MDA-MB-231 and (D) MDA-MB-468 cells was markedly impaired. "P $<0.05$ compared with that of the NC siRNA control; FOXCUT, forkhead box C1 promoter upstream transcript; NC, negative control; siRNA, small interfering RNA. 
Previous studies regarding BLBC-associated genes primarily focused on protein-coding genes. In recent years, numerous studies have demonstrated the involvement of lncRNAs in the development and progression of a number of types of malignant tumors (4-6). In breast cancers, several lncRNAs have been identified as novel biomarkers and therapeutic targets, including HOTAIR, BC200 and CCAT2 (21-23). However, the expression patterns and functional roles of cancer-associated lncRNAs in BLBCs remain to be determined.

The present study reported a novel lncRNA, FOXCUT, which may be a cancer-promoting gene responsible for the aggressive phenotype in BLBCs. Through RT-qPCR investigation, it was determined that the expression levels of IncRNA-FOXCUT were remarkably elevated in BLBCs, yet not in other non-basal like breast cancer subtypes. The expression levels of FOXCUT in BLBCs were significantly higher than those in non-basal like breast cancer subtypes, suggesting that IncRNA-FOXCUT may serve as a novel specific biomarker in BLBCs.

Furthermore, In BLBC cell lines, it was revealed that the knockdown of IncRNA-FOXCUT markedly inhibited cell proliferation and migration. This indicated that FOXCUT is not only a pure diagnostic marker in BLBCs, but also an important functional regulator in the cell aggressiveness, similar to the well-known lncRNA HOTAIR in breast cancer (21).

Cancer-associated lncRNAs may exert their regulating activities through diverse mechanisms. Certain lncRNAs may perform their functional roles by directly regulating their neighboring protein coding genes, such as lncRNA PVT1 and protein coding gene c-MYC $(24,25)$. FOXC1 (FOXCUT) is an adjacent IncRNA upstream of the FOXC1 promoter. These ncRNAs are called promoter upstream transcripts (PROMPTs) and are often functionally associated with the adjacent protein-coding transcripts (26-28). Given that the function of the protein-coding gene FOXC1 and FOXCUT lncRNA are involved in the progression of BLBCs by affecting the cell proliferation and cell migration (14-16), it is therefore speculated that FOXCUT lncRNA and FOXC1 mRNA may be another functional IncRNA-mRNA pair that interact with each other in BLBC. RT-qPCR results showed that the expression of FOXCUT lncRNA was positively correlated with FOXC1 mRNA. Through RNA interference analysis, it was determined that the knockdown of lncRNA-FOXCUT clearly reduced the levels of FOXC1 mRNA expression, which was in line with the inhibited cell growth rate and migration ability. This indicated that FOXCUT IncRNA may promote the aggressiveness of BLBC cells partly by regulating the expression of protein-coding gene FOXC1. However, additional studies are required for complete elucidation of the underlying mechanisms.

In conclusion, the present study was the first to identify the expression and functional role of a novel lncRNA, FOXCUT, and its association with the adjacent FOXC1 mRNA in BLBCs. The results indicated that FOXCUT may be a potential diagnostic marker and therapeutic target in the future.

\section{References}

1. Kreike B, van Kouwenhove M, Horlings H, et al: Gene expression profiling and histopathological characterization of triple-negative/basal-like breast carcinomas. Breast Cancer Res 9: R65, 2007.

2. Rakha EA, Reis-Filho JS and Ellis IO: Basal-like breast cancer: a critical review. J Clin Oncol 26: 2568-2581, 2008.
3. Carey LA, Perou CM, Livasy CA, et al: Race, breast cancer subtypes, and survival in the Carolina Breast Cancer Study. JAMA 295: 2492-2502, 2006.

4. Huarte M and Rinn JL: Large non-coding RNAs: missing links in cancer? Hum Mol Genet 19: R152-R161, 2010

5. Spizzo R, Almeida MI, Colombatti A and Calin GA: Long non-coding RNAs and cancer: a new frontier of translational research? Oncogene 31: 4577-4587, 2012.

6. Tsai MC, Spitale RC and Chang HY: Long intergenic noncoding RNAs: new links in cancer progression. Cancer Res 71: 3-7, 2011.

7. Okazaki Y, Furuno M, Kasukawa T, et al: Analysis of the mouse transcriptome based on functional annotation of 60,770 full-length cDNAs. Nature 420: 563-573, 2002.

8. Guttman M, Amit I, Garber M, et al: Chromatin signature reveals over a thousand highly conserved large non-coding RNAs in mammals. Nature 458: 223-227, 2009.

9. Perez DS, Hoage TR, Pritchett JR, et al: Long, abundantly expressed non-coding transcripts are altered in cancer. Hum Mol Genet 17: 642-655, 2008.

10. Han L, Zhang K, Shi Z, et al: LncRNA profile of glioblastoma reveals the potential role of IncRNAs in contributing to glioblastoma pathogenesis. Int J Oncol 40: 2004-2012, 2012.

11. Myatt SS and Lam EW: The emerging roles of forkhead box (Fox) proteins in cancer. Nat Rev Cancer 7: 847-859, 2007.

12. Xia L, Huang W, Tian D, et al: Overexpression of forkhead box $\mathrm{C} 1$ promotes tumor metastasis and indicates poor prognosis in hepatocellular carcinoma. Hepatology 57: 610-624, 2013.

13. Xu ZY, Ding SM, Zhou L, et al: FOXC1 contributes to microvascular invasion in primary hepatocellular carcinoma via regulating epithelial-mesenchymal transition. Int J Biol Sci 8: 1130-1141, 2012.

14. Sizemore ST and Keri RA: The forkhead box transcription factor FOXC1 promotes breast cancer invasion by inducing matrix metalloprotease 7 (MMP7) expression. J Biol Chem 287: 24631-24640, 2012.

15. Ray PS, Wang J, Qu Y, et al: FOXC1 is a potential prognostic biomarker with functional significance in basal-like breast cancer. Cancer Res 70: 3870-3876, 2010.

16. Ray PS, Bagaria SP, Wang J, et al: Basal-like breast cancer defined by FOXC1 expression offers superior prognostic value: a retrospective immunohistochemical study. Ann Surg Oncol 18: 3839-3847, 2011.

17. Wang L, Gu F, Liu CY, Wang RJ, Li J and Xu JY: High level of FOXC1 expression is associated with poor prognosis in pancreatic ductal adenocarcinoma. Tumour Biol 34: 853-858, 2013.

18. Wei LX, Zhou RS, Xu HF, Wang JY and Yuan MH: High expression of FOXC1 is associated with poor clinical outcome in non-small cell lung cancer patients. Tumour Biol 34: 941-946, 2013.

19. Livasy CA, Karaca G, Nanda R, et al: Phenotypic evaluation of the basal-like subtype of invasive breast carcinoma. Mod Pathol 19: 264-271, 2006.

20. Nielsen TO, Hsu FD, Jensen K, et al: Immunohistochemical and clinical characterization of the basal-like subtype of invasive breast carcinoma. Clin Cancer Res 10: 5367-5374, 2004.

21. Gupta RA, Shah N, Wang KC, et al: Long non-coding RNA HOTAIR reprograms chromatin state to promote cancer metastasis. Nature 464: 1071-1076, 2010.

22. Iacoangeli A, Lin Y, Morley EJ, et al: BC200 RNA in invasive and preinvasive breast cancer. Carcinogenesis 25: 2125-2133, 2004.

23. Redis RS, Sieuwerts AM, Look MP, et al: CCAT2, a novel long non-coding RNA in breast cancer: expression study and clinical correlations. Oncotarget 4: 1748-1762, 2013.

24. Shtivelman E and Bishop JM: Effects of translocations on transcription from PVT. Mol Cell Biol 10: 1835-1839, 1990.

25. Guan Y, Kuo WL, Stilwell JL, et al: Amplification of PVT1 contributes to the pathophysiology of ovarian and breast cancer. Clin Cancer Res 13: 5745-5755, 2007.

26. Preker P, Nielsen J, Kammler S, et al: RNA exosome depletion reveals transcription upstream of active human promoters. Science 322: 1851-1854, 2008.

27. Jacquier A: The complex eukaryotic transcriptome: unexpected pervasive transcription and novel small RNAs. Nat Rev Genet 10: 833-844, 2009.

28. Preker P, Almvig K, Christensen MS, et al: PROMoter uPstream Transcripts share characteristics with mRNAs and are produced upstream of all three major types of mammalian promoters. Nucleic Acids Res 39: 7179-7193, 2011. 